\title{
The Dietary Inflammatory Index Is Associated With Diabetes Severity
}

\author{
Dana E. King, MD, MS and Jun Xiang, MS, MA
}

Objective: The Dietary Inflammatory Index (DII) is a recently developed dietary inflammation assessment tool. The current study examined the association between DII and the presence and severity of diabetes in adults age $\geq 20$ years.

Research Design and Methods: Cross-sectional analysis of 4434 adult participants in the National Health and Nutrition Examination Survey (NHANES 2013 to 2014). The DII was calculated based on 24hour dietary recall data. Linear and logistic regression models were used to estimate the relationship and control for possible confounding factors.

Results: Among 4434 participants, mean age was 49.4 years, mean BMI (body mass index) was $29.3 \mathrm{~kg} /$ $\mathrm{m}^{2}$, and mean DII (higher is more inflammatory) was 0.65 (range, -3.41 to +9.05$)$. The mean DII scores in participants with and without diabetes were 0.79 and 0.50 , respectively $(P=.0098)$. Participants with Hemoglobin A1c (HgbA1c) >9\% had higher DII scores than those with $6.5 \%$ to $9 \%$ HgbA1c $(1.37$ vs $0.54, P=$ $.0002)$ and those with $<6.5 \%$ HgbA1c $(1.37$ vs $0.50, P<.0001)$. With 1 point increase in the DII score, odds of having diabetes increased by $13 \%(95 \%$ CI, 1.02 to 1.24). Among the individuals with diabetes, we also observed a significant association between severity of diabetes and DII scores; with 1 point increase in DII score, the odds of having HgbA1c higher than $9 \%$ increased by $43 \%$ (95\% CI, 1.21 to 1.68 ).

Conclusions: The DII had a significant association with diabetes and a stronger association when HgbA1c $>9 \%$. Further research will help clarify the association between inflammation and diet and the utility of the DII as a tool in risk assessment and management of patients with diabetes. (J Am Board Fam Med 2019;32:801-806.)

Keywords: Biomarkers, Body Mass Index, Cross-Sectional Studies, Diabetes Mellitus, Diet, Inflammation, Logistic Models, Nutrition Surveys, Risk Assessment

The Dietary Inflammatory Index (DII) has emerged as a possibly important tool in assessing diet quality and inflammation in the setting of high-

This article was externally peer reviewed.

Submitted 11 March 2019; revised 15 May 2019; accepted 18 May 2019.

From the Department of Family Medicine, West Virginia University, Morgantown, WV.

Funding: none.

Conflict of interest: none declared.

Author contributions: DK designed the study, wrote the manuscript, reviewed data and analysis, and researched references. JX wrote the manuscript, did the analysis, and researched references.

Research reported in this publication was supported in part by the National Institute of General Medical Sciences of the National Institutes of Health under Award Number 5U54GM104942-03. The content is solely the responsibility of the authors and does not necessarily represent the official views of the National Institutes of Health.

Corresponding author: Dana E. King, MD, MS, Department of Family Medicine, West Virginia University, Morgantown, WV 26505 (E-mail: kingdana@wvumedicine.org). risk medical conditions. The DII is a measure derived from analysis of multiple databases to measure the impact of 45 specific types of food on inflammatory biomarkers including IL-1 $\beta$, IL-4, IL-6, IL-10, TNF- $\alpha$, and C-reactive protein (CRP). ${ }^{1}$ Following the initial study that described the DII, subsequent studies have explored the association of the DII and a variety of chronic medical conditions, including chronic kidney disease, ${ }^{2}$ cardiovascular disease, ${ }^{3}$ depression, ${ }^{4}$ and metabolic syndrome. ${ }^{5}$

Diabetes has not been studied extensively in relation to the DII, but it represents an important medical condition that has been associated with inflammation ${ }^{6-9}$ and $\operatorname{diet}^{10-12}$ in a variety of studies. Determining further information about the DII and diabetes could facilitate its use in the clinical setting, and might provide a tool for the assessment of the risk of diabetes. However, there has been 
limited experience in studying the association of the DII and diabetes.

The goal of this study was to evaluate the relationship between DII and the presence of diabetes in a nationally representative sample of adults in the U.S population. A second goal was to explore the relationship between DII and the severity of diabetes.

\section{Methods}

\section{Study population}

The present study was a retrospective cross-sectional study using data from the continuous $\mathrm{Na}$ tional Health and Nutrition Examination Survey (NHANES 2013 to 2014). The NHANES is a series of complex and multistage surveys, conducted by the National Center for Health Statistics (NCHS), designed to assess the health and nutritional status of the noninstitutionalized US population. Since 1999, the continuous NHANES ${ }^{13}$ collected demographic, socioeconomic, dietary, and health-related information through 2 components, an in-home interview and a medical examination, on selected participants in 2-year cycles. Informed consents were obtained from all participants and the protocol for conducting the NHANES survey was approved by the NCHS Research Ethics Review Board. Details on survey design and response rate can be found on the NHANES Web site. ${ }^{13}$ Analyses for this study were limited to adults $\geq 20$ years of age (the customary classification cutoff in the NHANES) with nonmissing information for variables of interest. The NHANES uses $\geq 20$ years as the cutoff for adults, and we have used it to be consistent with many previous NHANES studies. The focus of the study was adults with diabetes because the role of inflammation in diabetes, while well established in adults, is not as well established in children. ${ }^{14}$ West Virginia University Institutional Review Board approved this study to be exempt.

\section{Definition of Nondiabetes, Prediabetes, Diabetes, and Severe Diabetes}

To define diabetes status of a participant, we followed the guideline from the American Diabetes Association using measured HgbA1c as a diagnostic criterion: without diabetes treatments, participants with HgbA1c less than $5.7 \%$, between $5.7 \%$ to $6.4 \%$, or $6.5 \%$ or greater would be categorized as having no diabetes, prediabetes, or diabetes respectively; $9 \%$ or greater HgbAlc would be defined as having severe diabetes. ${ }^{15} \mathrm{We}$ also added those people who answered positively to the question, "Were you told by a doctor that you have diabetes?" to identify additional individuals with diabetes.

\section{The DII and NHANES 2013 to 2014}

The DII is a tool, created to examine the inflammatory potential of individuals' diets. A description of the design and development of the original DII can be found elsewhere. ${ }^{1}$

The current study incorporated the latest version of DII, which represents an improved scoring algorithm based on extensive review of the literature and a world food consumption data from several countries. ${ }^{16}$ Briefly, a total of 45 food parameters (types of food and nutrients) derived from dietary data were assigned inflammatory effect scores based on the research findings from 1943 selected articles, examining the role of the food parameters on the 6 established inflammatory biomarkers (IL-1 $\beta$, IL-4, IL-6, IL-10, TNF-a, and C-reactive protein), published from 1950 to 2010. World food consumption data, based on 11 diverse populations around the world, was used to generate a mean and standard deviation for each food parameter. An individual's diet was then linked to the world food database as a z-score, calculated by subtracting the "standard global mean" and dividing its standard deviation. This z-score was then converted to a centered percentile score to minimize the risk of "right skewing." The product of the centered percentile score and the respective article generated inflammatory effect score for each food parameter was then summed to create an overall DII score for an individual. A total DII score could be positive or negative. Higher positive DII scores indicate more proinflammatory diets and more negative scores imply more anti-inflammatory diets.

In this study, we utilized a total of 28 out of the 45 food parameters, for which we had dietary intake data available from the 2 24-hour dietary recalls data in the NHANES 2013 to 2014 to calculate DII scores. These parameters include total calories, total fat, saturated fat, monounsaturated fat, polyunsaturated fat, omega-3 fatty acids, omega-6 fatty acids, protein, carbohydrate, fiber, alcohol, cholesterol, niacin, thiamin, vitamin A, vitamin B2, vitamin B6, vitamin B12, vitamin C, vitamin D, 
vitamin $\mathrm{E}$, iron, magnesium, selenium, zinc, folic acid, $\beta$ carotene, and caffeine. We calculated the total DII scores per 1000 calories of food consumed to control for the effect of different amounts of total energy intakes.

\section{Population Covariates}

We extracted population characteristics including age, gender, race, BMI (body mass index), physical activity, smoking status, alcohol use, and socioeconomic status (education level, health insurance status) as potential covariates. Age was divided into 3 groups: 20 to 44 years, 45 to 64 years, and 65 years and older. We examined race in 4 race groups of non-Hispanic white, non-Hispanic Black, Hispanic, and Other race, as they are categorized in the NHANES. ${ }^{13}$ There were 4 BMI categories combined as underweight $\left(<18.5 \mathrm{~kg} / \mathrm{m}^{2}\right)$, normal $(18.5$ to $24.9 \mathrm{~kg} / \mathrm{m}^{2}$ ), overweight ( 25 to $29.9 \mathrm{~kg} / \mathrm{m}^{2}$ ), and obese $\left(\geq 30 \mathrm{~kg} / \mathrm{m}^{2}\right)$ based on the Center for Disease Control and Prevention breakdown. Participants' education level was grouped into 2 categories of " $<$ High school" and " $\geq$ High school." Health insurance status was defined as "Yes" for having health insurance and "No" for not having health insurance. Two levels of physical activity were defined as " $\geq 150$ " or " $<150$ " minutes moderate-intense recreational physical activity per week. Smoking status was coded as "smoke" for current smokers and "not smoke" for current nonsmokers. For alcohol use, the cutoff for "alcohol use" and "no alcohol use" was at least 12 alcohol drinks in the past year.

\section{Statistical Analysis}

All analyses in this study were conducted using SAS (version 9.4, 2013, SAS Institute Inc. Cary, NC). To account for the complex survey design (including oversampling, survey nonresponse, and poststratification), we incorporated 2-year sampling weights and SAS survey analysis procedures following NHANES survey methods and analytic guidelines. $^{13}$

Population characteristics of the study sample were compared across diabetes status using $\chi^{2}$ test. Regression analyses were performed to determine the differences in DII scores between diabetes status and between the severity levels of diabetes. To examination the relationship between diabetes and DII scores adjusted for all covariates included age, sex, race, socioeconomic status, BMI categories, alcohol use, smoking status, and physical activity, we estimated multivariable adjusted odds ratios (ORs) using logistic regression models. There were no missing values for DII, and only 108 for HgbA1c. Missing values were addressed by the assumption of missing at random. All tests were 2 tailed, and P-values less than .05 were considered statistically significant.

\section{Results}

Based on the inclusion criteria, a total of 4434 subjects, $46.5 \%$ men and $53.5 \%$ women, were included from NHANES 2013 to 2014 for this study. The percentages of subjects with no diabetes, prediabetes, and diabetes were $59.4 \%, 26.5 \%$, and $14.1 \%$ respectively. As showed in Table 1 , subjects who were black, older, nonsmoker, not alcohol user, and having higher BMI, less education, health insurance, and less than 150 minutes exercise per week, were more likely to have diabetes.

Table 2 presents the comparison in mean DII scores between people with no diabetes, prediabetes, and with diabetes. Mean (SD) DII for the whole sample were 0.65 (1.50), with ranges between -3.41 to 9.05 (higher number is more inflammation). The least square means of DII scores for the no diabetes, prediabetes, and diabetes participants were $0.50,0.50$, and 0.79 , respectively. The results of the regression analyses indicated that subjects with diabetes had significantly higher DII scores than those without diabetes $(P=.01)$ and those with prediabetes $(P=.03)$. Among individuals with diabetes, those who had higher HgbAlc $(>9 \%)$ had higher DII scores than those with lower HgbA1c between $6.5 \%$ and $9 \%$ (1.37 vs $0.55, P=$ .0002).

After adjusting for age, sex, race, BMI, physical activity, smoking status, alcohol use, and socioeconomic status, we found a significant association between the incidence of diabetes and DII scores (Table 3). With 1 point increment in the DII score, odds of having diabetes increased by $13 \%$ (95\% CI, 1.02 to 1.24$)$.

We also examined severity of diabetes. Among the individuals with diabetes, we observed a significant association between severity of diabetes and DII scores, using a linear regression and continuous variables for HgbA1c and DII $(P<.04)$. For each 1-point increment in DII score, the odds of having HgbA1c higher than $9 \%$ increased by $43 \%$ 
Table 1. Population Characteristics by Diabetes Status in NHANES 2013 to $2014(\mathrm{~N}=4434)$

\begin{tabular}{|c|c|c|c|c|}
\hline Characteristic & Overall* & $\begin{array}{c}\text { No Diabetes }(\mathrm{n}=3810) \\
\%(95 \% \mathrm{CI})\end{array}$ & $\begin{array}{l}\text { Diabetes }(\mathrm{n}=624), \\
\%(95 \% \mathrm{CI})\end{array}$ & $P$-Value ${ }^{\dagger}$ \\
\hline \multicolumn{5}{|l|}{ Age (years) } \\
\hline 20 to 44 & 1862 & $96.4(95.2$ to 97.5$)$ & $3.6(2.5$ to 4.8$)$ & \multirow[t]{3}{*}{$<.0001$} \\
\hline 45 to 64 & 1562 & $86.0(83.3$ to 88.8$)$ & $14.0(11.2$ to 16.70 & \\
\hline $65+$ & 1010 & $78.8(76.6$ to 81.0$)$ & $21.2(19.0$ to 23.4$)$ & \\
\hline \multicolumn{5}{|l|}{ Sex, \% } \\
\hline Male & 2060 & $88.4(86.8$ to 89.9$)$ & $11.6(10.1$ to 13.2$)$ & \multirow[t]{2}{*}{.08} \\
\hline Female & 2374 & $89.9(88.6$ to 91.3$)$ & 10.1 (8.7 to 11.4$)$ & \\
\hline \multicolumn{5}{|l|}{ Race } \\
\hline Non-Hispanic white & 1988 & $90.0(88.7$ to 91.1$)$ & $10.0(8.8$ to 11.3$)$ & \multirow[t]{4}{*}{.0005} \\
\hline Non-Hispanic Black & 884 & $85.0(82.5$ to 87.4$)$ & $15.0(12.6$ to 17.5$)$ & \\
\hline Hispanic & 979 & $89.2(86.7$ to 91.7$)$ & $10.8(8.3$ to 13.3$)$ & \\
\hline Other & 583 & $88.4(85.7$ to 91.0$)$ & $11.6(9.0$ to 14.3$)$ & \\
\hline \multicolumn{5}{|l|}{ Education } \\
\hline$\geq$ High school & 3524 & 90.1 (88.8 to 91.4$)$ & 9.9 (8.6 to 11.2$)$ & \multirow[t]{2}{*}{$<.0001$} \\
\hline$<$ High school & 847 & $83.6(81.3$ to 86.0$)$ & $16.4(14.0$ to 18.7$)$ & \\
\hline \multicolumn{5}{|l|}{ Health insurance } \\
\hline Yes & 3533 & 88.3 (87.0 to 89.6$)$ & $11.7(10.4$ to 13.0$)$ & \multirow[t]{2}{*}{.0006} \\
\hline No & 896 & 93.4 (91.2 to 95.6$)$ & $6.6(4.4$ to 8.8$)$ & \\
\hline \multicolumn{5}{|l|}{ Physical activity } \\
\hline$\geq 150$ Minutes/week & 1400 & 93.2 (91.3 to 95.2$)$ & 6.7 (4.8 to 8.7$)$ & \multirow[t]{2}{*}{$<.0001$} \\
\hline$<150$ Minutes/week & 3034 & $87.0(86.0$ to 88.0$)$ & $13.0(11.9$ to 14.0$)$ & \\
\hline \multicolumn{5}{|l|}{ Body mass index } \\
\hline Underweight $\left(<18.5 \mathrm{~kg} / \mathrm{m}^{2}\right)$ & 68 & $97.9(94.7$ to 100$)$ & $2.1(0.0$ to 5.3$)$ & \multirow[t]{4}{*}{$<.0001$} \\
\hline Normal $\left(18.5\right.$ to $\left.24.9 \mathrm{~kg} / \mathrm{m}^{2}\right)$ & 1208 & $96.4(95.3$ to 97.5$)$ & $3.6(2.5$ to 4.7$)$ & \\
\hline Overweight ( 25 to $29.9 \mathrm{~kg} / \mathrm{m}^{2}$ ) & 1418 & 92.3 (91.0 to 93.6$)$ & $7.7(6.4$ to 9.0$)$ & \\
\hline Obese $\left(\geq 30 \mathrm{~kg} / \mathrm{m}^{2}\right)$ & 1707 & $81.3(79.3$ to 83.3$)$ & $18.7(16.7$ to 20.7$)$ & \\
\hline \multicolumn{5}{|l|}{ Smoking } \\
\hline Yes & 833 & $91.9(89.7$ to 91.2$)$ & $8.1(5.9$ to 10.3$)$ & \multirow[t]{2}{*}{.03} \\
\hline No & 3600 & $88.6(87.2$ to 90.0$)$ & $11.4(10.0$ to 12.8$)$ & \\
\hline \multicolumn{5}{|l|}{ Alcohol use } \\
\hline Yes & 3081 & 90.5 (89.6 to 91.4$)$ & 9.5 (8.6 to 10.4$)$ & \multirow[t]{2}{*}{$<.0001$} \\
\hline No & 1174 & $83.9(80.5$ to 87.4$)$ & $16.1(12.6$ to 19.5$)$ & \\
\hline
\end{tabular}

NHANES, National Health and Nutrition Examination Survey; CI, confidence interval.

*Unequal sample sizes due to missing values from subjects.

${ }^{\dagger} P$-value for comparison of difference in proportion between diabetic and non-diabetic subjects, using $\chi^{2}$ test.

(95\% CI, 1.21 to 1.68 ). When examining DII and HgbA1c as continuous variables, each 1 point increase in DII score was associated with an increase of 0.03 in $\mathrm{HgbA1c}(P=.04)$. There was negative correlation $(-0.57)$ between DII scores and dietary fiber intake.

\section{Discussion}

The results of the current study demonstrate a significant association between the DII and diabetes, and between the DII and severity of diabetes, with greater inflammation (higher DII) making diabetes and higher diabetes severity more likely.
The results remained significant after adjustment for possible confounders including age, sex, race, BMI, smoking status, alcohol use, physical activity, and socioeconomic status. The odds of having a HgbA1c higher than $9 \%$ increased by $43 \%$ with a $>1.0$ DII score toward a more inflammatory diet.

The study is consistent with previous literature on the association of inflammation and diabetes. ${ }^{6-9}$ Ridker and colleagues, ${ }^{6}$ for example, demonstrated that individuals with elevated levels of the inflammatory biomarker high-sensitivity CRP are at increased risk of mortality and morbidity from diabetes and other conditions, including myocardial 
Table 2. Means (Least Square) of Dietary Inflammatory Index (DII) Scores by Diabetes Status and Severity of Diabetes

\begin{tabular}{|c|c|c|c|}
\hline Diabetes Status & LS Mean & Std Error & $P$-Value \\
\hline No diabetes $(\mathrm{n}=2634)$ & $0.50^{8}$ & 0.05 & $.03^{\ddagger}$ \\
\hline Pre-diabetes $(\mathrm{n}=1176)$ & $0.50 \dagger$ & 0.06 & \\
\hline Diabetes $(n=624)$ & $0.79^{*} \dagger$ & 0.10 & \\
\hline \multicolumn{4}{|l|}{ Severity of Diabetes } \\
\hline Mild (HgbA1c $6.5 \%$ to $9 \%)(\mathrm{n}=375)$ & 0.55 & 0.11 & $.0002^{\S}$ \\
\hline Severe $(\operatorname{HgbA1c}>9 \%)(\mathrm{n}=94)$ & 1.37 & 0.14 & \\
\hline
\end{tabular}

The range of the DII scores was -3.41 to 9.05 . Positive scores are pro-inflammatory and negative scores are anti-inflammatory. ${ }^{*} P<.05$ for comparison of difference between non-diabetic and diabetic subjects.

${ }^{\dagger} P<.05$ for comparison of difference between pre-diabetic and diabetic subjects.

${ }^{\ddagger} P$-value for comparison of mean difference in DII scores between non-diabetic, pre-diabetic, and diabetic subjects using regression analysis.

${ }^{\S} P$-value for comparison of difference in DII scores between subjects with different level s of severity of diabetes using regression analysis.

Table 3. Odds Ratio (OR) and 95\% CI for the Relation Between the Diabetes Severity and Dietary Inflammatory Index (DII) Scores in NHANES 2013 to 2014

\begin{tabular}{lccc}
\hline Model & OR & $95 \%$ CI & $P$-Value* \\
\hline Model I & 1.13 & 1.02 to 1.24 & .02 \\
Model II & 1.43 & 1.21 to 1.68 & .0003 \\
Model $\mathrm{III}^{\S}$ & 1.37 & 1.27 to 1.46 & $<.0001$ \\
Model $\mathrm{IV}^{\text {Il }}$ & 0.99 & 0.85 to 1.15 & .85 \\
\hline
\end{tabular}

CI, confidence interval; DII, Dietary Inflammatory Index; NHANES, National Health and Nutrition Examination Survey. ${ }^{*} P$-values from logistic regression analysis for association between diabetes and DII scores, between severity of diabetes and DII scores, adjusted for age, sex, race, health insurance status, education level, BMI, smoking status, alcohol use, and physical activity.

${ }^{\dagger}$ Model I comparing diabetes to no diabetes.

${ }^{\ddagger}$ Model II comparing severe diabetes $(\mathrm{HgbA1c}>9)$ to mild diabetes $(6.5 \leq \mathrm{HgbA1} 1 \mathrm{c} \leq 9)$.

${ }^{\$}$ Model III comparing severe diabetes (HgbA1c > 9) to no diabetes.

${ }^{\mathbb{I} M o d e l}$ IV comparing mild diabetes $(6.5 \leq \mathrm{HgbA1c} \leq 9)$ to no diabetes.

infarction and stroke. King and colleagues ${ }^{8}$ previously showed in analysis of a national cohort, after controlling for age, race, sex, smoking, length of time with diabetes, insulin, and BMI, that HgbA1c was significantly associated with an increased likelihood of elevated CRP for HgbA1c >9.0\% (75 $\mathrm{mmol} / \mathrm{mol}$ ) (OR, 2.15; 95\% CI, 1.07 to 4.32). Such results support the findings of the current study that diet-induced inflammation (suggested by a dietary index based on self-reported intake) and diabetes are related, and that a more inflammatory diet profile is associated with a higher risk of severity of diabetes according to HgbA1c.

More recent studies have provided further support for the association. A recent British study investigated the cross-sectional association between an anti-inflammatory dietary pattern and diabetes in the National Diet and Nutrition Survey ${ }^{17}$. A total of 1531 survey members provided dietary data. A regression analysis was used to derive an anti-inflammatory dietary pattern. Overall, 52 survey members had diabetes. The derived anti-inflammatory pattern was inversely related to CRP, and was associated with lower odds of diabetes (adjusted OR for highest compared with lowest quintile: $0.17 ; 95 \%$ CI, 0.04 to 0.73 ). In research done by Koloverou and colleagues ${ }^{18}$, adherence to a low inflammatory Mediterranean diet was associated with a decreased risk of developing diabetes of $49 \%$ (95\% CI, 0.30 to 0.88 ) as well as lower levels of TNF- $\alpha$, CRP, and IL-6. Wholegrain cereals, fruits and legumes had the greatest predictive ability, which supports the current study finding of a high correlation of the DII and fiber intake $(-0.57)$.

The study has some limitations that should be considered. The time of diabetes diagnosis is not known for the cohort, and the study is cross-sectional, limiting the results to an association and not causality. Further, dietary intake data are limited to 2-day recall of intake, thus misclassification of DII could occur due to memory lapses or that the 24hour recall of diet is not representative of the person's overall diet. However, 24-hour recall of di- 
etary intake as a method of collecting diet history is considered a reasonable estimate for populations. ${ }^{19}$ In addition, one third of the DII parameters were missing from the NHANES database. However, the DII is based on a global database of foods, while the foods included in the NHANES are the common foods consumed in the United States. The strengths of the study include a national sample and the consistency of results when controlling for possible confounding factors.

In conclusion, the implications of the current study are consistent with previous research regarding the importance of inflammation in the diet as a factor in diabetes and its severity. A higher DII score for higher inflammation was associated with a higher likelihood of diabetes and severe diabetes ( $>9 \%$ HgbA1c). Further research is needed to determine whether the DII tool could be useful in practice, and whether a diet that specifically targets the DII parameters could be used to reduce the development of diabetes or its severity.

The authors thank Ashley Higinbotham, West Virginia University Medicine, for help with preparing the manuscript.

To see this article online, please go to: http://jabfm.org/content/ 32/6/801.full.

\section{References}

1. Cavicchia PP, Steck SE, Hurley TG, et al. A new dietary inflammatory index predicts interval changes in serum high-sensitivity C-reactive protein. J Nutr 2009;139:2365-2372.

2. Mazidi M, Shivappa N, Wirth MD, et al. Greater Dietary Inflammatory Index score is associated with higher likelihood of chronic kidney disease. $\mathrm{Br} \mathrm{J}$ Nutr 2018;120:204-209.

3. Park SY, Kang M, Wilkens LR, et al. The Dietary Inflammatory Index and All-Cause, Cardiovascular Disease and Cancer Mortality in the Multiethnic Cohort Study. Nutrients 2018;10(12). pii: E1844.

4. Bergmans RS, Malecki KM. The association of dietary inflammatory potential with depression and mental well-being among U.S. adults. Prev Med 2017;99:313-319.

5. Camargo-Ramos CM, Correa-Bautista JE, CorreaRodriguez M, et al. Dietary Inflammatory Index and cardiometabolic risk parameters in overweight and sedentary subjects. Int J Environ Res Public Health 2017;14(10). pii: E1104.
6. Ridker PM. Inflammatory biomarkers and risks of myocardial infarction, stroke, diabetes, and total mortality: implications for longevity. Nutr Rev 2007; 65(12 Pt 2):S253-S2539.

7. Calle MC, Fernandez ML. Inflammation and type 2 diabetes. Diabetes Metab 2012;38:183-191.

8. King DE, Mainous AG 3rd, Buchanan TA, et al. C-reactive protein and glycemic control in adults with diabetes. Diabetes Care 2003;26:1535-1539.

9. Brahimaj A, Ligthart S, Ghanbari M, et al. Novel inflammatory markers for incident pre-diabetes and type 2 diabetes: the Rotterdam Study. Eur J Epidemiol 2017;32:217-226.

10. Knowler WC, Fowler SE, Hamman RF, et al. 10year follow-up of diabetes incidence and weight loss in the Diabetes Prevention Program Outcomes Study. Lancet 2009;374:1677-1686.

11. Tonstad S, Butler T, Yan R, et al. Type of vegetarian diet, body weight, and prevalence of type 2 diabetes. Diabetes Care 2009;32:791-796.

12. Della Pepa G, Vetrani C, Vitale M, et al. Wholegrain intake and risk of type 2 diabetes: evidence from epidemiological and intervention studies. $\mathrm{Nu}-$ trients 2018;10(9). pii: E1288.

13. National Center for Health Statistics (NCHS). NHANES Survey methods and analytic guidelines. Available from: https://wwwn.cdc.gov/nchs/nhanes/ analyticguidelines.aspx. Accessed October 2018.

14. Reinehr T, Roth CL. Inflammation markers in type 2 diabetes and the metabolic syndrome in the pediatric population. Curr Diab Rep 2018;18:131.

15. Standards of medical care in diabetes-2018 abridged for primary care providers. Available from: http:// clinical.diabetesjournals.org/content/36/1/14. Accessed October 2018.

16. Shivappa N, Steck SE, Hurley TG, et al. Designing and developing a literature-derived, populationbased dietary inflammatory index. Public Health Nutr 2014;17:1689-1696.

17. McGeoghegan L, Muirhead CR, Almoosawi S. Association between an anti-inflammatory and antioxidant dietary pattern and diabetes in British adults: results from the national diet and nutrition survey rolling programme years $1-4$. Int J Food Sci Nutr 2015; 67(5):553-61.

18. Koloverou E, Esposito K, Gugliano D, Panigiotakos D. The effect of Mediterranean diet on the development of type 2 diabetes mellitus: a meta-analysis of 10 prospective studies and 136,846 participants. Metabolism 2014; 63(7):903-11.

19. Ortiz-Andrellucchi A, Sánchez-Villegas A, DoresteAlonso J, et al. Dietary assessment methods for micronutrient intake in elderly people: a systematic review. Br J Nutr 2009;102(Suppl 1):S118-S1149. 